\title{
The Role and Potential Application of Antimicrobial Peptides in Autoimmune Diseases
}

\author{
Chunye Zhang ${ }^{1}$ and Ming Yang ${ }^{2 *}$ \\ ${ }^{1}$ Department of Veterinary Pathobiology, University of Missouri, Columbia, MO, United States, ${ }^{2}$ Department of Surgery, \\ University of Missouri, Columbia, MO, United States
}

Keywords: autoimmune disease, antimicrobial peptides, immunomodualtion, therapy, diagnostic marker

\section{INTRODUCTION}

Most of the antimicrobial peptides (AMPs) are small, cationic, and amphipathic peptides with $<50$ amino acids (1), which are also known as host defense peptides (HDPs). As listed in the Antimicrobial Peptide Database (APD) (2), over 2300 AMPs have been identified from animals. Above 130 AMPs have been tested in humans to date. They are constitutively and inducibly expressed in a variety of tissues where pathogens easily access, including gastrointestinal, urinary

OPEN ACCESS

Edited by:

Attila Mócsai,

Semmelweis University, Hungary

Reviewed by:

Janine Adele Lamb,

University of Manchester,

United Kingdom

Eddie A. James,

Benaroya Research Institute,

United States

*Correspondence:

Ming Yang

yangmin@health.missouri.edu

Specialty section:

This article was submitted to Autoimmune and Autoinflammatory

Disorders,

a section of the journal

Frontiers in Immunology

Received: 10 February 2020 Accepted: 15 April 2020

Published: 08 May 2020

Citation:

Zhang C and Yang M (2020) The Role and Potential Application of

Antimicrobial Peptides in Autoimmune

Diseases. Front. Immunol. 11:859.

doi: 10.3389/fimmu.2020.00859 and respiratory tracts, and epithelial surfaces (3). Cathelicidins and defensins are two groups of AMPs broadly studied in humans. Defensins can be further subclassified into $\alpha$-defensins and $\beta$-defensins by their secondary structures. AMPs have pleiotropic functions. The most wellknown function is the antimicrobial activity against invading microorganisms, including bacteria, fungi, and enveloped viruses (4-6). Moreover, AMPs display other biological functions, such as lipopolysaccharide (LPS)-neutralization, wound healing, chemotactic activity, and immune modulation (3, 7-9). All their physiochemical characteristics, such as the molecular size, net charge, secondary or three-dimensional structure, and hydrophobicity, together determine their biological functions $(7,10,11)$.

An occurrence of autoimmune diseases (ADs) happens when the host immune system attacks self-tissues with complex pathogenesis. The etiologies causing ADs are not fully understood. The genome-wide association studies (GWAS) reveal that genetic risk factors are involved in different ADs such as rheumatoid arthritis (RA), multiple sclerosis (MS), systemic lupus erythematosus (SLE), Type 1 diabetes (T1D), and celiac disease (12). The development of ADs in geneticallysusceptible individuals is often triggered by environmental factors, including infectious agents, chemicals, and diets (13). The accumulating evidence shows that AMPs also play pivotal roles in autoimmune disorders. Most of the AMPs are induced or upregulated in the development of ADs, indicating their potential side effects (Figure 1A). For example, human cathelicidin LL-37 is positively associated with the pathogenesis of psoriasis, RA, and SLE $(14,15)$.

Microorganisms inhabit different areas, such as the intestine, skin, and oral sites. Bacteria, as the main part of microbes, have been considered to play a vital role in the pathogenesis of ADs at those organs (16). For instance, Manfredo Vieira et al. found that translocation of a gut bacterium Enterococcus gallinarum to the liver or other organs can trigger autoimmune responses in mice with a genetic background of predisposing to lupus-like autoimmunity (17). Recent metagenomics studies also reveal that dysbiosis of gut microbiota impacts the progression of ADs in infants $(18,19)$. In addition, dysbiosis of gut microbiota induced by environmental factors is able to change the expression of AMPs, which indicates the crosslink between gut microbiota and ADs. Herein, in this review, we discuss the potential effects of AMPs in different ADs and their potential roles as diagnostic and therapeutic agents. 


\section{A}

\begin{tabular}{|c|c|c|}
\hline Autoimmune diseases & Antimicrobial peptides & Expression \\
\hline Crohn's disease (CD) & Human $\alpha$-defensins 5 (HD5) & Upregulated \\
\hline Multiple sclerosis (MS) & Defensins (HDs and HBDs) & Upregulated \\
\hline Psoriasis & $\begin{array}{c}\text { Human cathelicidin LL-37 } \\
\text { Human } \beta \text {-defensin 2 (HBD-2) }\end{array}$ & Upregulated \\
\hline $\begin{array}{c}\text { Rheumatic arthritis (RA) } \\
\text { Systemic lupus erythematosus } \\
\text { (SLE) }\end{array}$ & $\begin{array}{c}\text { Human } \beta \text {-defensin 3 (HBD3), } \\
\text { Rat cathelicidin related antimicrobial peptide (rCRAMP) }\end{array}$ & Upregulated \\
\hline Type 1 diabetes (T1D) & $\begin{array}{c}\text { Human } \beta \text {-defensin 2 (HBD2) } \\
\text { Human neutrophil peptides (HNPs) }\end{array}$ & Upregulated \\
\hline
\end{tabular}

B Infection, injury, genetics Proliferation
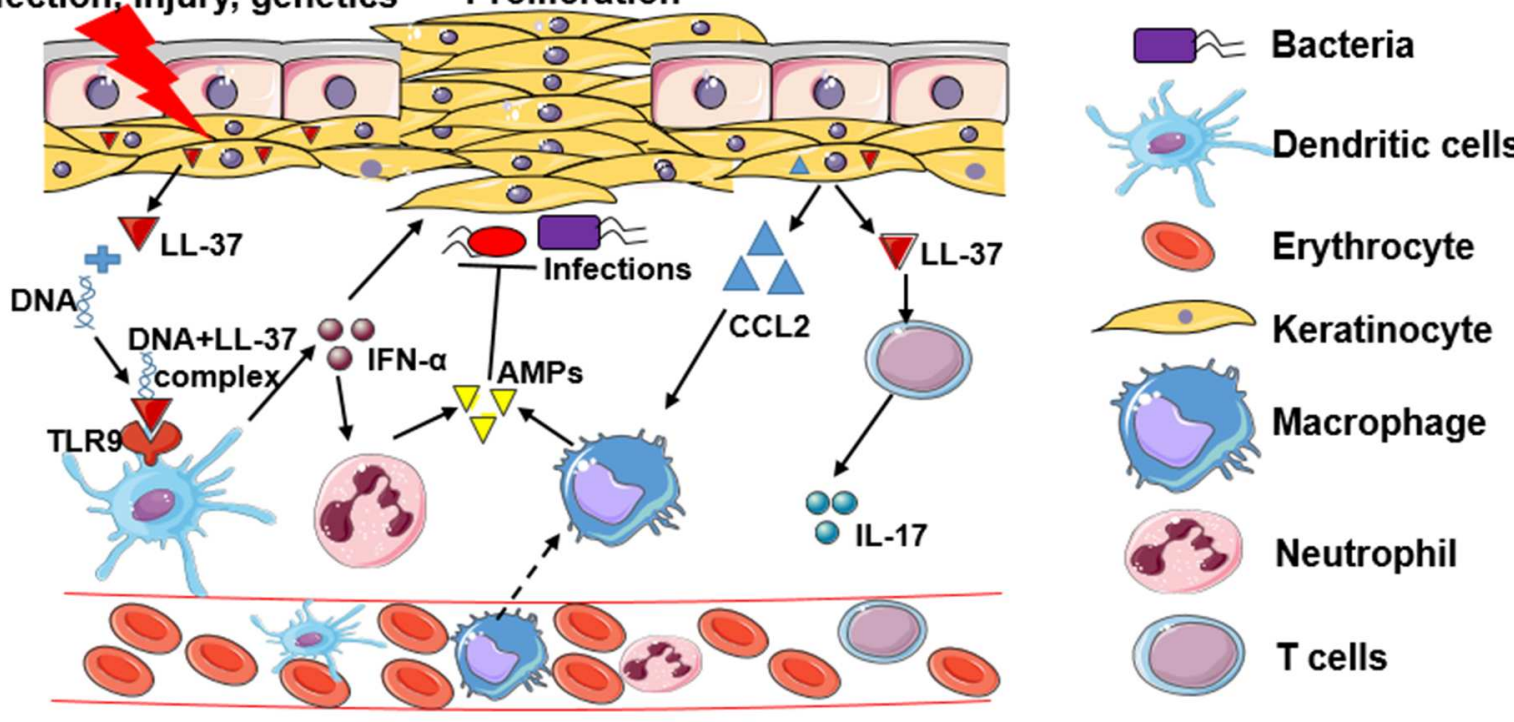

FIGURE 1 | The role of antimicrobial peptides in autoimmune diseases and the molecular mechanism of human cathelicidin LL-37 in psoriasis. (A) Examples of antimicrobial peptides (AMPs) upregulated in different autoimmune diseases (ADs). (B) The molecular mechanism of human cathelicidin LL-37 in psoriasis. Under the pressure of infection, injury, or genetics, keratinocytes are activated to express high levels of cathelicidin LL-37, which binds with non-stimulatory self-DNA to form the self-DNA-LL-37 complexes to activate plasmacytoid dendritic cells (pDCs) to produce interferons (e.g., IFN- $\alpha$ ) in a TLR9-dependent manner. IFN- $\alpha$ can not only stimulate the proliferation of keratinocytes but can stimulate the activation of neutrophils to express more AMPs. In addition, LL-37, as an autoantigen, can activate T cells to express cytokines (e.g., IL-17), which also stimulate the proliferation of keratinocytes. Chemokines (e.g., CCL2) expressed in keratinocytes, and other immune cells further induce monocyte or macrophage migration to promote inflammatory responses. In this process, LL-37 and other AMPs also have antimicrobial activity.

\section{THE POTENTIAL UTILITY OF ANTIMICROBIAL PEPTIDES AS BIOMARKERS FOR AUTOIMMUNE DISEASES}

AMPs have displayed multiple roles in the innate and adaptive immunity. Accumulating evidence suggests that AMPs could be diagnostic markers for ADs. For instance, the expression of human neutrophil peptides (HNPs) 1-3 significantly increased in the synovial cavity of patients with RA (20). The significant correlation between the joint erosion and the presence of HNPs suggests they might be used as diagnostic markers for RA. In addition, the plasma level of HNP 1-3 was significantly increased in T1D patients compared to healthy controls (21). Serum levels of HBD-2 and HNPs are also elevated in SLE patients (22). Cathelicidin LL-37 and autoantibodies to LL-37 were elevated in patients with psoriatic arthritis (PA), which was correlated with clinical inflammatory markers (23). In the following context, we will describe the role of AMPs in different ADs and how AMPs function in their development. 


\section{PSORIASIS}

Psoriasis is a chronic autoimmune skin disease resulting from genetic, epigenetic, environmental, and lifestyle factors (24), which affects 125 million people worldwide, according to the World Psoriasis Day consortium (https://ifpa-pso.com/ouractions/world-psoriasis-day). Aberrant production of interferons (IFNs) in plasmacytoid dendritic cells (pDCs) is a major pathogenic effector in psoriasis, which triggers the activation of $\mathrm{T}$ cells. As shown in Figure 1B, antimicrobial peptide LL-37 secreted by keratinocytes of lesional skin binds with non-stimulatory self-DNA to form the self-DNA-antimicrobial peptide complexes. These complexes can translocate into pDCs to activate IFN- $\alpha$ production in a TLR9-dependent manner (25), which promotes the progression of psoriasis. LL-37 also can bind with self-RNA from dying cells to form self-RNALL-37 complexes, which can trigger the secretion of IFN- $\alpha$ in pDCs by activating TLR7 and TLR8 to exacerbate psoriasis (26). Lande et al. also reported that LL-37 could directly trigger $\mathrm{T}$ cell activation as an autoantigen in psoriasis (27). In addition to keratinocytes, increased infiltrating neutrophils are another group of cells that primarily produce AMPs in psoriasis. Meanwhile, neutrophils can induce human $\beta$-defensin 2 (HBD2) expression in keratinocytes of psoriatic skin by forming neutrophil extracellular traps (NETs) (28). Except for neutrophils and pDCs, other AMP-producing innate lymphocytes such as natural killer T (NKT) cells and natural killer (NK) cells also play critical roles in the pathogenesis of psoriasis (29). Overall, AMPs with AMP-producing cells play an essential role in the pathogenesis of psoriasis through both the innate and adaptive immune responses.

\section{RHEUMATIC ARTHRITIS}

Inflammatory arthritis (IA) is an autoimmune disease characterized by synovial hyperplasia, which can be driven by genetic and environmental factors. The proteomics and peptidomics analysis of synovial fluid (SF) showed that AMPs played an essential role in the molecular underpinnings of IA (30). For example, the expression of human $\alpha$-defensin 3 (HD-3) is significantly upregulated in the SF of IA patients. RA is the most common and destructive IA, a well-known chronic autoimmune disorder (31). Neutrophils contribute to the pathogenesis of RA by secreting pro-inflammatory cytokines and chemokines, forming NET, and generating autoantigens to drive tissue damage (32). The rat cathelicidin related antimicrobial peptide (rCRAMP) was significantly upregulated in the joints of rats with pristane-induced arthritis (PIA) (33). The expression of rCRAMP was primarily from neutrophils and macrophages (14). In the SF of human RA patients, $\mathrm{CD}_{163}{ }^{+}$macrophages and $\mathrm{CD}^{+} 6^{+}$granulocytes are the primary cells expressing LL-37, which has been discussed to display a boosting effect in psoriasis. On the other side, treatment with anti-rheumatic agents, such as adalimumab and etanercept, caused a significant reduction of LL-37 expression, which indicates that there is an association between the expression of LL-37 and RA disease severity (34).

\section{TYPE 1 DIABETES}

Type 1 diabetes (T1D) is an autoimmune disease resulting from the destruction of insulin-producing pancreatic $\beta$-cells (35). The upregulation of cathelicidin related antimicrobial peptide (CRAMP) in the intestine of diabetes-prone BioBreeding (BBdp) rats was observed during the development of diabetes. The gut microbiota and dietary antigens impact the incidence of T1D both in animals and humans $(36,37)$. Gut microbiota can control the expression of CRAMP in pancreatic $\beta$-cells through the production of short-chain fatty acids (38). These studies indicate that modulating gut microbiota can be applied to reduce the development of autoimmune diabetes through the change of AMP expression.

\section{THE ROLE OF GUT MICROBIOTA IN OTHER AUTOIMMUNE DISEASES}

In addition to T1D, dysbiosis of gut microbiota may also trigger other autoimmune diseases, such as MS and SLE (39, 40). Emerging evidence shows that probiotic treatment may improve the prognosis of autoimmune diseases by modulating gastrointestinal symptoms and multi-organ inflammation (41). One mechanism of efficacy of probiotic therapy is to modulate the secretion of AMPs in the intestine $(42,43)$. Several other approaches, including AMPs, antibiotics, fecal microbiota transplantation (FMT), and prebiotics, have been applied to regulate the gut microbiota in clinical or preclinical studies to treat autoimmune disorders (44). Overall, dysbiosis of gut microbiota may trigger $\mathrm{ADs}$ in genetically susceptible persons, and modulating gut microbiota by antimicrobial intervention can be applied to prevent the development of ADs.

\section{THE BENEFICIAL ROLE OF ANTIMICROBIAL PEPTIDES IN AUTOIMMUNE DISEASES}

AMPs bridge the connection of innate and adaptive immune responses with a broad spectrum of microbicidal activity. Hence, they are double-edged swords in autoimmune disorders. In addition to the above-mentioned side effect, exogenous AMPs treatment can reduce the symptoms of $\mathrm{AD}$. For instance, administration of scolopendrasin IX, an AMP that was identified from centipede Scolopendra, could inhibit the expression of inflammatory cytokines through formyl peptide receptor 2 (FPR2), such as tumor necrosis factor alpha (TNF- $\alpha$ ) and interleukin 6 (IL-6). Administration of scolopendrasin IX also can reduce the clinical phenotype of RA in a mouse model induced by transgenic K/BxN mice serum (45). Treatment with rhesus $\theta$-defensin 1 (RTD-1), a family of macrocyclic peptides exclusively expressed in monkey, suppressed the joint disease progression and restored limb mobility in a rat model of rheumatoid arthritis (46). Furthermore, the in vivo treatment with the LL-37 peptide in BBdp rats promoted $\beta$-cell neogenesis and upregulation of potentially beneficial gut microbes, resulting in a reduction of symptoms of autoimmune diabetes (36). 
Injection of mouse $\beta$-defensin-14 (mBD14) ameliorated the central nervous system inflammation and pro-inflammatory cytokines and cytotoxic $\mathrm{T}$ cells in the model of experimental autoimmune encephalomyelitis. In vitro study further showed that human $\beta$-defensin-3, the human ortholog of mBD14, shifted non-regulatory $\mathrm{CD} 4{ }^{+} \mathrm{CD} 25^{-} \mathrm{T}$ cells into a regulatory phenotype with the expression of Treg markers, such as Foxp3 and GARP (47). Overall, these research results indicate that there are some beneficial effects of AMPs in AD treatment.

\section{SUMMARY}

Pro-inflammatory cytokines and chemokines play a pivotal role in the pathogenesis of autoimmune diseases (48). The exposure of pro-inflammatory cytokines, such as TNF- $\alpha$ and LPS, could significantly increase the expression of HBD-2 in cultured chondrocytes (49). Furthermore, AMPs secreted from activated chondrocytes, keratinocytes, or neutrophils can further impose the inflammatory response by chemoattracting more monocytes or other immune cells, advancing tissue damage manifested in patients with $\mathrm{ADs}$ (50). The loop of initial proinflammatory response-AMP expression-immune cell infiltration plays a crucial role in the progression of autoimmune disorders. Recently, gut microbiota has been shown to play a key role in the pathogenesis of ADs. For instance, lower

\section{REFERENCES}

1. Mahlapuu M, Hakansson J, Ringstad L, Bjorn C. Antimicrobial peptides: an emerging category of therapeutic agents. Front Cell Infect Microbiol. (2016) 6:194. doi: 10.3389/fcimb.2016.00194

2. Wang G, Li X, Wang Z. APD3: the antimicrobial peptide database as a tool for research and education. Nucleic Acids Res. (2016) 44:D1087-93. doi: $10.1093 /$ nar/gkv1278

3. Wang G. Human antimicrobial peptides and proteins. Pharmaceuticals. (2014) 7:545-94. doi: 10.3390/ph70 50545

4. McKelvey JA, Yang M, Jiang Y, Zhang S. Salmonella enterica serovar enteritidis antimicrobial peptide resistance genes aid in defense against chicken innate immunity, fecal shedding, and egg deposition. Infect Immunity. (2014) 82:5185-202. doi: 10.1128/IAI.02387-14

5. Yang M, Zhang C, Zhang MZ, Zhang S. Beta-defensin derived cationic antimicrobial peptides with potent killing activity against gram negative and gram positive bacteria. BMC Microbiol. (2018) 18:54. doi: 10.1186/s12866-018-1190-Z

6. Yang $M$, Zhang $C$, Zhang X, Zhang MZ, Rottinghaus GE, Zhang S. Structure-function analysis of Avian beta-defensin- 6 and beta-defensin12: role of charge and disulfide bridges. BMC Microbiol. (2016) 16:210. doi: 10.1186/s12866-016-0828-y

7. Yang M. Avian $\beta$-Defensins as Antimicrobial and Immunomodulatory Agents (2019). Available online at: https://hdl.handle.net/10355/68953

8. Yang M, Zhang C, Hansen SA, Mitchell WJ, Zhang MZ, Zhang S. Antimicrobial efficacy and toxicity of novel CAMPs against $P$. aeruginosa infection in a murine skin wound infection model. BMC Microbiol. (2019) 19:293. doi: 10.1186/s12866-019-1657-6

9. Zhang C, Yang M, Ericsson AC. Antimicrobial peptides: potential application in liver cancer. Front Microbiol. (2019) 10:1257. doi: 10.3389/fmicb.2019.01257

10. Wimley WC. Describing the mechanism of antimicrobial peptide action with the interfacial activity model. ACS Chem Biol. (2010) 5:905-17. doi: $10.1021 / \mathrm{cb} 1001558$
Firmicutes/Bacteroidetes ratio has been shown in SLE (39). Meanwhile, changes in the gut and oral bacteria are implicated in the pathogenesis of other ADs, such as RA, systemic sclerosis, and anti-phospholipid syndrome. Modulations of gut microbiota through probiotics or fecal transplantation (FMT) are considered a promising therapeutic strategy for ADs (39). AMPs, as the product and modulator of gut microbiota, can also be applied to defense against the infection and balance the dysbiosis of gut microbiota, which is beneficial to the treatment of ADs. Currently, the roles of AMPs in the pathogenesis of autoimmune diseases are better understood. The clinical trial results for investigation of AMPs in autoimmune disorders are expected. A combination of AMPs with other non-aggressive biomarkers (e.g., TNF- $\alpha$ ) could improve the diagnosis of autoimmune disorders in the early stage. Additionally, AMPs are promising targets to design personalized treatment for patients with ADs.

\section{AUTHOR CONTRIBUTIONS}

$\mathrm{CZ}$ and MY conceived the opinion and wrote the manuscript.

\section{FUNDING}

The study was supported by the Postdoctoral Research Grant of University of Missouri, Columbia, Missouri, USA.
11. Yang $M$, Zhang C, Zhang MZ, Zhang S. Novel synthetic analogues of avian beta-defensin-12: the role of charge, hydrophobicity, and disulfide bridges in biological functions. BMC Microbiol. (2017) 17:43. doi: 10.1186/s12866-017-0959-9

12. Lettre G, Rioux JD. Autoimmune diseases: insights from genomewide association studies. Hum Mol Genet. (2008) 17:R116-121. doi: $10.1093 / \mathrm{hmg} / \mathrm{ddn} 246$

13. Costenbader KH, Gay S, Alarcon-Riquelme ME, Iaccarino L, Doria A. Genes, epigenetic regulation and environmental factors: which is the most relevant in developing autoimmune diseases? Autoimmun Rev. (2012) 11:604-9. doi: 10.1016/j.autrev.2011.10.022

14. Hoffmann MH, Bruns H, Backdahl L, Neregard P, Niederreiter B, Herrmann $\mathrm{M}$, et al. The cathelicidins LL-37 and rCRAMP are associated with pathogenic events of arthritis in humans and rats. Annal Rheumatic Dis. (2013) 72:123948. doi: 10.1136/annrheumdis-2012-202218

15. Moreno-Angarita A, Aragón CC, Tobón GJ. Cathelicidin LL-37: a new important molecule in the pathophysiology of systemic lupus erythematosus. J Transl Autoimmun. (2020) 3:100029. doi: 10.1016/j.jtauto.2019.100029

16. Ruff WE, Kriegel MA. Autoimmune host-microbiota interactions at barrier sites and beyond. Trends Mol Med. (2015) 21:233-44. doi: 10.1016/j.molmed.2015.02.006

17. Manfredo Vieira S, Hiltensperger M, Kumar V, Zegarra-Ruiz D, Dehner C, Khan N, et al. Translocation of a gut pathobiont drives autoimmunity in mice and humans. Science. (2018) 359:1156-61. doi: 10.1126/science.aar7201

18. Kostic AD, Gevers D, Siljander H, Vatanen T, Hyotylainen T, Hamalainen AM, et al. The dynamics of the human infant gut microbiome in development and in progression toward type 1 diabetes. Cell Host Microbe. (2015) 17:260-73. doi: 10.1016/j.chom.2015.01.001

19. Russell JT, Roesch LFW, Ordberg M, Ilonen J, Atkinson MA, Schatz DA, et al. Genetic risk for autoimmunity is associated with distinct changes in the human gut microbiome. Nat Commun. (2019) 10:3621. doi: 10.1038/s41467-019-11460-x

20. Bokarewa MI, Jin T, Tarkowski A. Intraarticular release and accumulation of defensins and bactericidal/permeability-increasing protein in patients with rheumatoid arthritis. J Rheumatol. (2003) 30:1719-24. 
21. Nemeth BC, Varkonyi T, Somogyvari F, Lengyel C, Fehertemplomi $\mathrm{K}$, Nyiraty S, et al. Relevance of alpha-defensins (HNP1-3) and defensin beta-1 in diabetes. World J Gastroenterol. (2014) 20:9128-37. doi: 10.3748 /wjg.v20.i27.9128

22. Vordenbaumen S, Fischer-Betz R, Timm D, Sander O, Chehab G, Richter J, et al. Elevated levels of human beta-defensin 2 and human neutrophil peptides in systemic lupus erythematosus. Lupus. (2010) 19:1648-53. doi: $10.1177 / 0961203310377089$

23. Frasca L, Palazzo R, Chimenti MS, Alivernini S, Tolusso B, Bui L, et al. Anti-LL37 antibodies are present in psoriatic arthritis (PsA) patients: new biomarkers in PsA. Front Immunol. (2018) 9:1936. doi: 10.3389/fimmu.2018.01936

24. Albanesi C, Madonna S, Gisondi P, Girolomoni G. The interplay between keratinocytes and immune cells in the pathogenesis of psoriasis. Front Immunol. (2018) 9:1549. doi: 10.3389/fimmu.2018.01549

25. Lande R, Gregorio J, Facchinetti V, Chatterjee B, Wang Y-H, Homey B, et al. Plasmacytoid dendritic cells sense self-DNA coupled with antimicrobial peptide. Nature. (2007) 449:564-9. doi: 10.1038/nature06116

26. Ganguly D, Chamilos G, Lande R, Gregorio J, Meller S, Facchinetti $\mathrm{V}$, et al. Self-RNA-antimicrobial peptide complexes activate human dendritic cells through TLR7 and TLR8. J Exp Med. (2009) 206:1983-94. doi: $10.1084 /$ jem. 20090480

27. Lande R, Botti E, Jandus C, Dojcinovic D, Fanelli G, Conrad C, et al. The antimicrobial peptide LL37 is a T-cell autoantigen in psoriasis. Nat Commun. (2014) 5:5621. doi: 10.1038/ncomms6621

28. Hu SC-S, Yu H-S, Yen F-L, Lin C-L, Chen G-S, Lan C-CE. Neutrophil extracellular trap formation is increased in psoriasis and induces human $\beta$ defensin-2 production in epidermal keratinocytes. Sci Rep. (2016) 6:31119. doi: $10.1038 /$ srep31119

29. Polese B, Zhang H, Thurairajah B, King IL. Innate lymphocytes in psoriasis. Front Immunol. (2020) 11:242. doi: 10.3389/fimmu.2020. 00242

30. Mahendran SM, Keystone EC, Krawetz RJ, Liang K, Diamandis EP, Chandran V. Elucidating the endogenous synovial fluid proteome and peptidome of inflammatory arthritis using label-free mass spectrometry. Clin Proteom. (2019) 16:23. doi: 10.1186/s12014-0199243-3

31. Jokar M, Jokar M. Prevalence of inflammatory rheumatic diseases in a rheumatologic outpatient clinic: analysis of 12626 cases. J Rheumatol Res. (2018) 3:21-7. doi: 10.22631/rr.2017.69997.1037

32. O'Neil LJ, Kaplan MJ. Neutrophils in rheumatoid arthritis: breaking immune tolerance and fueling disease. Trends Mol Med. (2019) 25:215-27. doi: 10.1016/j.molmed.2018.12.008

33. Hoffmann MH, Herrmann M, Agerberth B, Holmdahl R. The antimicrobial peptide rCRAMP is strongly upregulated during experimental arthritis in the rat. Annals Rheumat Dis. (2012) 71:A29. doi: 10.1136/annrheumdis-2011-201232.9

34. Neregård P, Engström M, Lindh M, Agerberth B, Catrina AI. AB0100 identification of the antimicrobial peptide LL-37 as a potential mediator of synovial inflammation in RA. Annals Rheumat Dis. (2013) 71:643. doi: 10.1136/annrheumdis-2012-eular.100

35. Katsarou A, Gudbjörnsdottir S, Rawshani A, Dabelea D, Bonifacio E, Anderson BJ, et al. Type 1 diabetes mellitus. Nat Rev Dis Primers. (2017) 3:17016. doi: $10.1038 /$ nrdp. 2017.16

36. Pound LD, Patrick C, Eberhard CE, Mottawea W, Wang G-S, Abujamel T, et al. Cathelicidin antimicrobial peptide: a novel regulator of islet function, islet regeneration, and selected gut bacteria. Diabetes. (2015) 64:4135. doi: $10.2337 / \mathrm{db} 15-0788$

37. Thomas RM, Jobin C. Microbiota in pancreatic health and disease: the next frontier in microbiome research. Nat Rev Gastroenterol Hepatol. (2020) 17:53-64. doi: 10.1038/s41575-019-0242-7

38. Sun J, Furio L, Mecheri R, van der Does AM, Lundeberg E, Saveanu L, et al. Pancreatic beta-cells limit autoimmune diabetes via an immunoregulatory antimicrobial peptide expressed under the influence of the gut microbiota. Immunity. (2015) 43:304-17. doi: 10.1016/j.immuni.2015.07.013

39. De Luca F, Shoenfeld Y. The microbiome in autoimmune diseases. Clin Exp Immunol. (2019) 195:74-85. doi: 10.1111/cei.13158

40. de Oliveira GLV, Leite AZ, Higuchi BS, Gonzaga MI, Mariano VS. Intestinal dysbiosis and probiotic applications in autoimmune diseases. Immunology. (2017) 152:1-12. doi: 10.1111/imm.12765

41. Liu Y, Alookaran JJ, Rhoads JM. Probiotics in autoimmune and inflammatory disorders. Nutrients. (2018) 10:1537. doi: 10.3390/nu101 01537

42. Al-Ghazzewi FH, Tester RF. Impact of prebiotics and probiotics on skin health. Benef Microb. (2014) 5:99-107. doi: 10.3920/BM2013.0040

43. Bermudez-Brito M, Plaza-Diaz J, Munoz-Quezada S, Gomez-Llorente C, Gil A. Probiotic mechanisms of action. Annals Nutrition Metabol. (2012) 61:160-74. doi: 10.1159/000342079

44. Balakrishnan B, Taneja V. Microbial modulation of the gut microbiome for treating autoimmune diseases. Expert Rev Gastroenterol Hepatol. (2018) 12:985-96. doi: 10.1080/17474124.2018.1517044

45. Park YJ, Park B, Lee M, Jeong YS, Lee HY, Sohn DH, et al. A novel antimicrobial peptide acting via formyl peptide receptor 2 shows therapeutic effects against rheumatoid arthritis. Sci Rep. (2018) 8:14664. doi: 10.1038/s41598-018-32963-5

46. Schaal JB, Tran DQ, Subramanian A, Patel R, Laragione T, Roberts KD, et al. Suppression and resolution of autoimmune arthritis by rhesus $\theta$-defensin- 1 , an immunomodulatory macrocyclic peptide. PLoS ONE. (2017) 12:e0187868. doi: 10.1371/journal.pone. 0187868

47. Bruhs A, Schwarz T, Schwarz A. Prevention and mitigation of experimental autoimmune encephalomyelitis by murine $\beta$-defensins via induction of regulatory T cells. J Invest Dermatol. (2016) 136:173-81. doi: 10.1038/JID.2015.405

48. Moudgil KD, Choubey D. Cytokines in autoimmunity: role in induction, regulation, and treatment. J Interferon Cytokine Res. (2011) 31:695-703. doi: 10.1089/jir.2011.0065

49. Varoga D, Pufe T, Harder J, Meyer-Hoffert U, Mentlein R, Schroder JM, et al. Production of endogenous antibiotics in articular cartilage. Arthrit Rheum. (2004) 50:3526-34. doi: 10.1002/art.20605

50. Froy O, Sthoeger ZM. Defensins in systemic lupus erythematosus. Ann N Y Acad Sci. (2009) 1173:365-9. doi: 10.1111/j.1749-6632.2009.04622.x

Conflict of Interest: The authors declare that the research was conducted in the absence of any commercial or financial relationships that could be construed as a potential conflict of interest.

Copyright (c) 2020 Zhang and Yang. This is an open-access article distributed under the terms of the Creative Commons Attribution License (CC BY). The use, distribution or reproduction in other forums is permitted, provided the original author(s) and the copyright owner(s) are credited and that the original publication in this journal is cited, in accordance with accepted academic practice. No use, distribution or reproduction is permitted which does not comply with these terms. 